\title{
Determinants of Optimum Breastfeeding Among Mothers of Child Less than Two Years in Bishoftu Town, East Shewa Zone of Oromia Region, Ethiopia
}

\author{
Zelalem Kebede \\ Public Health Program, Police Medical Professionals Training Institute, Ethiopian Police University College, Addis Abeba, Ethiopia
}

Email address:

welzol@yahoo.com

To cite this article:

Zelalem Kebede. Determinants of Optimum Breastfeeding Among Mothers of Child Less than Two Years in Bishoftu Town, East Shewa Zone of Oromia Region, Ethiopia. Science Journal of Public Health. Vol. 3, No. 4, 2015, pp. 544-551. doi: 10.11648/j.sjph.20150304.23

\begin{abstract}
Back ground - The promotion and support of breastfeeding is a global priority. Vast scientific literatures demonstrated substantial health, social and economic benefits associated with appropriate breastfeeding, including lower infant morbidity and mortality from diarrhea and infectious diseases. WHO and UNICEF recommend that all mothers should breastfeed their children exclusively for the first 6 months and thereafter. In Ethiopia, like in other developing nations, diarrhea is a major contributor of morbidity and mortality in young infant and children, especially in urban areas, due to inappropriate breastfeeding patterns. Breastfeeding being a vital and indispensable part of primary health care, studies have not been conducted in East Shewa zone on determinants of optimum breastfeeding among mothers of reproductive age group. Objective - To assess determinants of optimum breastfeeding among mothers of child aged less than two years in Bishoftu town, Ada'a woreda, East Shewa zone of Oromia Regional State, Ethiopia. Methods - A cross- sectional community based study design was employed. A total of 806 mothers of children age less than two years residing in Bishoftu town, East Shewa zone, Ethiopia were selected by using systematic random sampling for the quantitative study. The households were selected by systematic random sampling, which is by dividing the total number of households in all selected kebeles by the allocated sample size to get study subjects. Experienced interviewers and qualified supervisors were employed and a pre-tested questionnaire was utilized for this purpose. Result—Four hundred ten (50.9\%) had sufficient knowledge on benefit of breastfeeding. Knowledge was found to be influenced by PNC. The prevalence of exclusive breastfeeding practice is only $34.1 \%$. PNC follow up and maternal educations are significantly associated with exclusive breastfeeding practice. Mothers who attended PNC are more likely to practice exclusive breastfeeding than those who did not attend ( $\mathrm{OOR}=2.19(1.12,4.32)$ ), and those who did not attend formal school are more likely to practice exclusively $(\mathrm{AOR}=3.61(1.14,11.43))$, than mothers who attended education. Conclusion: It is concluded that maternal knowledge on benefit of breastfeeding is insufficient and breastfeeding practice is sub-optimal. This would increase the risk of faltered growth, infant/young child diarrhea and/or malnutrition, ARI and in addition it shortens birth interval and high fertility. Therefore strengthening counseling mothers on optimal breastfeeding practice and improving the information provision by suitable focused intensive IEC activity and other recommendations is forwarded.
\end{abstract}

Keywords: Breastfeeding, Exclusive Breastfeeding, Determinant Factors

\section{Introduction}

The promotion and support of breastfeeding is a global priority (1). Vast scientific literatures demonstrated substantial health, social and economic benefits associated with appropriate breast feeding, including lower infant morbidity and mortality from diarrhea and infectious diseases (1)

Benefits of breastfeeding to the child include: a decrease of the incidence and/or severity of infectious diseases such as diarrhea, respiratory tract infections, otitis media and urinary tract infection; decreased incidence of types 1 and 2 diabetes mellitus, overweight, obesity, and asthma. $(1,2)$

Breastfeeding also increases the maternal-infant bonding. In the long run, it may decrease the risk of breast and ovarian cancers, and osteoporosis in the mother (3)

WHO and UNICEF recommend that all mothers should breastfeed their children exclusively for the first 6 months 
and thereafter. They should continue to breastfeed for as long as the mother and child wish, and both appropriate and sufficient weaning food should be added after six months of life $(4,5)$. WHO estimates that worldwide only $35 \%$ of children between birth and their fifth month are breastfed exclusively (6).

Though it is now widely accepted that breast milk is the best for the baby, it is also a well-known fact that exclusive breastfeeding rates in early infancy are still too low (1-3). A number of studies have shown that deficits in knowledge amongst health workers (3, 6, and 7) and lack of adequate information being given to mothers (8-11) are major factors responsible for low rates of exclusive breastfeeding. Maternal education, race and socio-economic factors are also known to influence breastfeeding decisions $(8,12,13)$.

In Ethiopia, like in other developing nations, diarrhea is a major contributor of morbidity and mortality in young infant and children, especially in urban areas, due to inappropriate breastfeeding patterns (7). About $58 \%$ of deaths in children are associated with malnutrition. About $70 \%$ of infant are sub-optimally breastfed, with current $24 \%$ of infant death due to poor breastfeeding practice (8).

EDHS of the 2005 indicates that only $49 \%$ of infants were exclusively breastfed with reception of prelacteal feed in $29 \%$ of newborn and only $69 \%$ of them were put to breast within one hour (9).

A study conducted in Addis Ababa revealed that, $60 \%$ of mothers provided pre-lacteal fluids and $32 \%$ of infants less than four months timely complimentary feeding rate were $57.4 \%(10)$

Breastfeeding being a vital and indispensable part of primary health care, studies have not been conducted in EastShoa zone on determinants of optimum breastfeeding among mothers of reproductive age group

Therefore the purpose of this study is to investigate determinants of breastfeeding among women of child under two years of children who reside in Bishoftu town and will have a significant input in the formulation of adjustments strategies that should be considered to increase optimum breastfeeding practices.

\section{Methods}

\subsection{Study Design, Period \& Area}

A cross-sectional community based study design was employed among mothers of child less than two years preceding the survey in Bishoftu town between May \& June 2010 .

The study was conducted in Bishoftu town, found in Ada'a Woreda, East Shewa zone of Oromia Regional State. Bishoftu is located to the east of the capital Addis Ababa at about $47 \mathrm{Kms}$. The town is divided in to nine kebeles and as projected from census 2007, the town has a total population of 100,114 in 2009.

The town has one hospital, one health center, several other private clinics and limited numbers of non-governmental health facilities.

\subsection{Study Population}

All mothers of children aged 0-23 months residing in Bishoftu town. The proportion of children less than two years constitutes $2.1 \%$ of the total population (14). Therefore the required sample sizes from each kebeles was calculated from sampling frame produced from all kebeles according to the size of population using proportionate to population size.

\subsection{Variables}

Optimum breastfeeding, maternal knowledge on advantage of breastfeeding, Exclusive breastfeeding were dependent variables. Socio-demographic and other health service related characteristics, such as maternal age, education, religion, ethnicity, marital and occupational status, ANC, PNC, \& Place of delivery were the independent variables.

\subsection{Sampling Technique}

Assuming that prevalence of $50 \%$ is taken since breastfeeding rate in the area is not known, $95 \%$ confidence interval, $(\alpha=0.05)$, the degree of precision to be $5 \%, 5 \%$ nonresponse rate and using single population proportion formula $(\mathrm{N}=\mathrm{z} 2 \mathrm{p}(1-\mathrm{p}) / \mathrm{d} 2)$ a sample size of 384 was calculated. Considering non response rate of $5 \%$, it will be 403 and since kebeles are selection units which are clusters, requiring adjustment for a design effect of $2 \%$ the total sample size that will be used for the study is 806 mothers of child age less than two years. A two stage sampling technique was used in sampling the study subjects. Bishoftu town was selected by convenience. All the nine kebeles of the town were included in the sampling frame. The total population of all kebeles of the town is 100,114 out of which 6877 are estimated to be children less than two years of age. It is from these target population that the required sample size was taken proportionately according to the size of the sampling frame in each kebeles.

\subsection{Data Collection}

A structured questionnaire was developed in English and translated to the Amharic language. The Amharic version was used for collecting information after introduction to data collectors and supervisors and was tested in the field just after training. Some extra questions were added and some were amended after training and field practice of the questionnaire. Trained data collectors were used to collect data on the variables from mothers of child aged two years and less for 10 days. A supervisor was responsible for 4 data collectors and collected filled questionnaires checked every day for inconsistencies and omissions. Submission of filled formats to coordinator was made every day, which was rechecked for any problem. Formats with problem were sent back to supervisors for re interview. To ensure the external validity (generalizability) of the study, appropriate size and representative type of study units were selected as described in the appropriate section above. And to ensure the internal 
validity (accuracy and precision) of the study, maximum effort was applied to minimize bias and errors.

The quality of data was controlled at different levels for completeness and consistency; first by data collectors at the end of each day, then by supervisors every day, then by the investigator, and finally during data entry. The investigator undertook computer data entry, cleaning and edition. The inconsistent and missed questionnaires were not found thus none were excluded from analysis.

\subsection{Scoring}

Summary score was calculated for knowledge of benefit of breast feeding and knowledge adequacy of breastfeeding based on 8 knowledge related questions. The mean score for benefit of breastfeeding knowledge was taken as a cut-off point and those who scored above the mean were considered as having sufficient knowledge, while those below the mean score labeled as having insufficient knowledge for the benefit of breast feeding.

\subsection{Data Processing and Analysis}

The collected data was cleaned for completeness and consistencies. Responses in each question were coded for simplicity of data entry. Then, $100 \%$ of the data was entered to EPI INFO version 6.0 package and transported to SPSS version 15.0 for analysis.

Descriptive statistics was used to determine the frequency of different variables. Results were presented using tables and graphs and interpreted as significant at a p-value of $<0.05$ and OR $95 \%$ CI.

Chi square test was used to detect the association between different variables contributing to optimum breastfeeding. To measure the strength of association for each significantly associated explanatory variable to the dependent variable, bivariate analysis is used. Finally, by multivariate analysis possible confounders are controlled.

\subsection{Ethical Consideration}

Ethical clearance was obtained from AAU, Medical Faculty. Letters was written to Bishoftu town administration and other concerned bodies to obtain permission and cooperation for data collection. Study participant were briefed about the confidentiality of their responses and the importance of providing the right information. Informed verbal consent was secured from the study subjects to participate in the study.

\section{Results}

\subsection{Socio-Demographic Characteristics}

A total of 806 mothers with children less than 2 years of age voluntarily responded, making the response rate $100 \%$. The mean age of the mothers was 26 years $(S D \pm 5.63)$ and ranges from 16 to 45 years. The age range of children considered in this study was $0-23$ months which is an optimal recommended age range for breastfeeding. The mean age of the children was 11.67 months $\left(\mathrm{SD}_{-}+6.79\right)$. Male children were $445(55.2 \%)$ and male to female ratio was 1.23: 1 .

\subsection{Knowledge on Benefits of Breastfeeding}

Mothers were assessed whether they have adequate knowledge about the benefits of breast feeding or not. Benefits of breastfeeding such as contraceptive effect, nutritive importance, disease protection, and mother to child bonding and other related advantages were also mentioned in different ways to ascertain the level of mothers' knowledge. The mean score for benefit of breastfeeding knowledge was found to be 5.331(SD+1.90) and those who scored above the mean were considered as having sufficient knowledge, while those below the mean score labeled as having insufficient knowledge for the benefit of breast feeding. Based on this assumption, only 410 (50.9\%) mothers have sufficient knowledge about the benefits of breast feeding for both mother and child. Maternal knowledge was positively associated with PNC follow up. Mothers who attended Postnatal care are more likely to have sufficient knowledge $(\mathrm{AOR}=1.53(1.12,2.10)$ as compared to those who had not have follow up. In addition mothers were assessed whether they have adequate knowledge about the advantage of breastfeeding specifically on contraceptive effect, and the three criteria of LAM or not. It was found that, out of all eligible mothers only $1 \%$ had knowledge of the recommended criteria.

\subsection{Exclusive Breastfeeding}

The two extreme age group mothers practiced exclusive breastfeeding as compared to other age groups. On the other hand no sex differences in proportion of the exclusive breastfeeding practice among male and female babies (Table 2). After an adjustment was made using logistic regression, exclusive breastfeeding was associated significantly with postnatal care. Mothers who did not attend formal school practiced exclusive breastfeeding more likely $(\mathrm{AOR}=3.61$ $(1.14,10.5))$ than who attended it, and respondents who have visited health institution for postnatal care during their last pregnancy, practiced exclusive breastfeeding more likely than those who did not attended it, $(\mathrm{AOR}=2.19$ (1.12 and 4.32) (Table 2).

\subsection{Optimal Breastfeeding Practice}

Optimal breastfeeding duration is recommended for minimum time of 2 Years. Having this indication, mothers of children aged one and above year were asked whether they have continued breastfeeding for the optimal duration of one year and above or not. It was found that out of 328, 206 $(62.8 \%)$ mothers of children $>12$ months of age were breastfeeding for $>12$ months. In this study, statistically significant association was not revealed between optimal breastfeeding duration and socio-demographic variables and other maternal health service related variables. Optimal breastfeeding practice criteria is included in the Strategy for 
Children Survival in Ethiopia, as exclusive breast feeding practice for the first six months, timely complementary feeding at the age of 6 months and continue breastfeeding at least 2 years. Having these criteria mothers in this study are categorized as having good practice if they practice the above feeding pattern optimally, and those who did not, as having poor practice.

Based on this assumption $48(8.8 \%)$ have good practice while $498(91.2 \%)$ mothers have poor practice about breast feeding. Mothers aged 15-19 years have good practice as compared to other age groups. The study shows a statistically significant negative relationship between optimal breastfeeding practice and occupational status. Working mothers were found to have less practice of optimal breastfeeding $(\mathrm{AOR}=0.28(0.11,0.74))$ than mothers who stayed at home. On the other hand the study revealed positive relationship between average family income and optimal breastfeeding practice. Those mothers with the average house hold income of less than 100ETB (AOR=3.11 $(1.11,8.74))$ were found to practice optimal breastfeeding significantly more likely than those who earn $>500$ ETB (Table 3 ).

Table 1. Association of selected socio-demographic and health service related variables with maternal knowledge towards the benefits of breastfeeding, Bishoftu town, Ethiopia, 2009

\begin{tabular}{|c|c|c|c|c|}
\hline \multicolumn{5}{|c|}{ Knowledge about advantage of breastfeeding $(n=806)$} \\
\hline Variables & Sufficient $\mathbf{N}^{\mathbf{0}}(\%)$ & Insufficient $\mathrm{N}^{0}(\%)$ & Crude OR (95\% CI) & Adjusted OR (95\% CI) \\
\hline \multicolumn{5}{|l|}{ Religion } \\
\hline Christian & $383(51.7)$ & $358(48.3)$ & $1.62(.96,2.71)$ & $1.70(1.01,2.87)$ \\
\hline Muslims & $26(40)$ & $39(60)$ & 1.00 & 1.00 \\
\hline \multicolumn{5}{|c|}{ Attended formal school } \\
\hline No & $83(48.5)$ & $88(51.5)$ & $0.89(.63,1.25)$ & $0.97(.68,1.38)$ \\
\hline Yes & $327(51.5)$ & $308(48.5)$ & 1.00 & 1.00 \\
\hline \multicolumn{5}{|c|}{ Mother's occupation } \\
\hline Employed & $128(52.9)$ & $114(47.1)$ & $1.13(.80,1.58)$ & $1.07(.79,1.46)$ \\
\hline Not employed & $282(50)$ & $282(50)$ & 1.00 & 1.00 \\
\hline \multicolumn{5}{|l|}{ ANC follow up } \\
\hline Yes & $387(51.4)$ & $366(48.6)$ & $1.38(.79,2.45)$ & $1.17(.65,2.01)$ \\
\hline No & $23(43.4)$ & $30(56.6)$ & 1.00 & 1.00 \\
\hline \multicolumn{5}{|l|}{ PNC follow up } \\
\hline Yes & $305(54.3)$ & $257(45.7)$ & $* 1.57(1.16,2.13)$ & $* 1.53(1.12,2.10)$ \\
\hline No & $105(43.3)$ & $139(56.7)$ & 1.00 & 1.00 \\
\hline \multicolumn{5}{|l|}{ Place of birth } \\
\hline Home & $67(45)$ & $82(55)$ & $0.75(.52,1.07)$ & $.80(.55,1.17)$ \\
\hline Health facility & $343(51.4)$ & $314(48.6)$ & 1.00 & 1.00 \\
\hline
\end{tabular}

Significant at $* \mathrm{P}=0.003$

$\mathrm{OR}=$ Odds ratio; $\mathrm{CI}=$ Confidence Interval; $\mathrm{ANC}=$ Antenatal Care; $\mathrm{PNC}=$ Postnatal Care.

Table 2. Association of selected Socio-demographic and health service related variables among mothers of child aged less than 6 months with exclusive breastfeeding, Bishoftu town, Ethiopia, 2009

\begin{tabular}{|c|c|c|c|c|}
\hline \multicolumn{5}{|c|}{ Exclusive breastfeeding $(n=205)$} \\
\hline Variables & Yes № $(\%)$ & No No $(\%)$ & Crude OR (95\% CI) & Adjusted OR (95\% CI) \\
\hline \multicolumn{5}{|l|}{ Mothers age } \\
\hline $15-19$ & $6(40.4)$ & $9(60.0)$ & $1.58(.34,7.22)$ & $4.47(.36,5.39)$ \\
\hline $20-24$ & $20(31.7)$ & $43(68.3)$ & $1.43(.48,4.29)$ & $1.45(.41,5.11)$ \\
\hline $25-29$ & $23(34.8)$ & $45(65.2)$ & $1.31(.44,3.89$ & $1.04(.3,3.61)$ \\
\hline $30-34$ & $14(33.8)$ & $28(66.2)$ & $1.40(.44,4.46)$ & $1.58(.42,5.9)$ \\
\hline \multicolumn{5}{|c|}{ Attended formal school } \\
\hline No & $21(37.5)$ & $35(62.5)$ & $* 3.75(1.25,11.3)$ & *3.61(1.14,10.5) \\
\hline Yes & $49(32.9)$ & $100(67.1)$ & 1.00 & 1.00 \\
\hline \multicolumn{5}{|c|}{ Mothers occupation } \\
\hline Employed & $19(29.2)$ & $66(70.8)$ & $1.39(.74,2.62)$ & $0.92(.42,2.03)$ \\
\hline Not employed & $51(36.4)$ & $89(63.6)$ & 1.00 & 1.00 \\
\hline \multicolumn{5}{|l|}{ ANC follow up } \\
\hline Yes & $64(33.6)$ & $126(66.4)$ & $1.68(1.61,2.40)$ & $0.41(.09,4.61)$ \\
\hline No & $69(40.0)$ & $9(60.0)$ & 1.00 & 1.00 \\
\hline \multicolumn{5}{|l|}{ PNC follow up } \\
\hline Yes & $37(46.2)$ & $43(53.8)$ & *2.39(1.33,4.34) & *2.19(1.12,4.32 \\
\hline No & $33(33.5)$ & $92(65.5$ & 1.00 & 1.00 \\
\hline
\end{tabular}




\begin{tabular}{|c|c|c|c|c|}
\hline \multicolumn{5}{|c|}{ Exclusive breastfeeding $(n=205)$} \\
\hline Variables & Yes № $(\%)$ & No № $(\%)$ & Crude OR (95\% CI) & Adjusted OR (95\% CI) \\
\hline \multicolumn{5}{|l|}{ Place of birth } \\
\hline Outside health facility & $7(41.2)$ & $10(58.8)$ & $0.76(.26,2.23)$ & $0.89(.29,2.74)$ \\
\hline Health facility & $63(33.5)$ & $125(65.5)$ & 1.00 & 1.00 \\
\hline
\end{tabular}

Significant at $* \mathrm{p}<0.05$

$\mathrm{OR}=$ Odds ratio; $\mathrm{CI}=$ Confidence Interval; $\mathrm{ANC}=$ Antenatal Care; $\mathrm{PNC}=$ Postnatal Care

Table 3. Association of selected socio-demographic and health service related variables among mothers of children aged 6-23 months with Optimum Breastfeeding practice, Bishoftu town, Ethiopia, 2009

\begin{tabular}{|c|c|c|c|c|}
\hline \multicolumn{5}{|c|}{ Optimum Breastfeeding practice $(n=546)$} \\
\hline Variables & Good No (\%) & Poor No (\%) & Crude OR (95\% CI) & Adjusted OR (95\% CI) \\
\hline \multicolumn{5}{|l|}{ Mother's age } \\
\hline $15-19$ & $6(18.7)$ & $26(81.3)$ & $1.06(0.2,5.59)$ & $0.61(0.59,6.30)$ \\
\hline $20-24$ & $12(10.3)$ & 113(89.7) & $1.59(0.58,4.36)$ & $1.61(0.48,5.43)$ \\
\hline 25-29 & $14(9.0)$ & $160(91.0)$ & $1.38(0.52,3.67)$ & $1.69(0.53,5.49)$ \\
\hline $30-34$ & $10(8.6)$ & $116(91.4)$ & $1.31(0.47,3.69)$ & $1.67(0.49,5.36$ \\
\hline$\geq 35$ & $6(6.7)$ & $83(93.3)$ & 1.00 & 1.00 \\
\hline \multicolumn{5}{|l|}{ Religion } \\
\hline Christians & $43(8.7)$ & $454(91.3)$ & $1.07(0.37,3.11)$ & $0.93(0.31,2.83$ \\
\hline Muslims & $5(10.2)$ & $44(89.8)$ & 1.00 & 1.00 \\
\hline \multicolumn{5}{|c|}{ Attended formal school } \\
\hline Yes & 13(11.5) & $100(88.5)$ & $1.48(0.75,2.89)$ & $0.78(0.35,1.75)$ \\
\hline No & $35(8.1)$ & 398(91.9) & 1.00 & 1.00 \\
\hline \multicolumn{5}{|c|}{ Mother's occupation } \\
\hline Employed & $6(3.8)$ & 153(96.2) & $* 0.32(0.13,0.77)$ & $* 0.28(0.11,0.74)$ \\
\hline Not employed & $42(10.9)$ & $345(89.1)$ & 1.00 & 1.00 \\
\hline \multicolumn{5}{|c|}{ Average income } \\
\hline $0-100$ & $9(20.9)$ & $34(79.1)$ & $* * 3.37(1.29,8.76)$ & $* * 3.11(1.11,8.74)$ \\
\hline $101-200$ & $9(9.1)$ & $90(90.9)$ & $1.27(0.51,3.19)$ & $0.94(0.35,2.50)$ \\
\hline $201-500$ & $14(4.9)$ & $270(95.1)$ & $1.05(0.46,2.36)$ & $0.76(0.31,1.73)$ \\
\hline$>500$ & $15(9.6)$ & 104(90.4) & 1.00 & 1.00 \\
\hline \multicolumn{5}{|l|}{ ANC follow up } \\
\hline Yes & $42(8.3)$ & 464(91.7) & $0.51(0.2,1.29)$ & $0.39(0.14,1.06)$ \\
\hline No & $6(15.0)$ & $34(85)$ & 1.00 & 1.00 \\
\hline \multicolumn{5}{|l|}{ PNC follow up } \\
\hline Yes & $32(9.1)$ & $318(90.9)$ & $1.13(0.61,2.12)$ & $1.60(0.79,3.24)$ \\
\hline No & $16(8.2)$ & $180(91.8)$ & 1.00 & 1.00 \\
\hline
\end{tabular}

Significant at $* \mathrm{p}=0.01, * * \mathrm{p}=0.03$

\section{Discussion}

This major purpose of this study aims at identifying major factors influencing optimum breastfeeding including maternal knowledge, health and biomedical constraints, attitudinal variables and program methods and to accordingly forward appropriate recommendations.

Accordingly key findings were maternal knowledge influenced by whether the mother attended PNC or not $(\mathrm{AOR}=1.53(1.12,2.10)$, exclusive breastfeeding whether the mother attended formal school or not $(\mathrm{AOR}=3.61(1.14,10.5)$ and optimum breastfeeding influenced both by maternal occupation and average household income with AOR of $0.28(0.11,0.74) \& 3.11(1.11,8.74)$ respectively.

\subsection{Knowledge on Benefits of Breastfeeding}

This study showed that out of all respondents $410(50.9 \%)$ have satisfactory knowledge on the benefit of breastfeeding both to mother and baby. However, the result is much lower than a study done in Jimma which is $67.2 \%(15)$. This could be explained by the fact that mothers might not be well informed about IYCF both during their visits to health facilities and at community level. Moreover health staffs and community worker/agencies might not be well trained on the issue IYCF so that they will be able to provide adequate information on how mothers should breastfeed, introduce timely complimentary foods and optimally breastfeeding as well. Postnatal attendance of mothers to health facilities 
showed significant association with maternal knowledge. Those who attended PNC had sufficient knowledge of breastfeeding $(\mathrm{AOR}=1.53(1.12,2.10))$ compared to those who didn't attend and this is consistent with a similar study done in Jimma (AOR=2.5) (15)

About 1\% mothers had knowledge about the three criteria of LAM. This is much lower than the assessment done in Madagascar, $18 \%$ mothers of age less than six months and $13 \%$ mothers of age $6-23$ months knew the three criteria of LAM (16), and 2005 EDHS showed, nationally, 8.9\% currently married women had the knowledge of LAM (9).

This could be explained by the fact that health workers in the area might lack familiarity with this method and thus the exclusion of LAM from modern method of contraception and accordingly the provision of information about this method.

This study revealed that $97.3 \%$ of the study subject ever breastfed at some point of time. It is consistent with the findings of 2005 EDHS, (96.0\%), and study done in Jimma $(98.8 \%)(9,32)$, and with the recent study done in Tigray, $(99.1 \%)(17)$. On the other hand about $2.7 \%$ of the study subjects have never breastfed. The majority cited reason were maternal problem, which include sickness; nipple problems and few did not want to respond.

In our study $71.8 \%$ mothers of children $<12$ months initiate breastfeeding within one hour after birth. This result is much higher than the result of study done in Madagascar $(50.0 \%)(16)$ and is consistent with the result of 2005 EDHS, $69.1 \%, 66.2 \%$ nationally and Addis Ababa respectively, and study done in Jimma $64.0 \%(9,15)$ and community assessment done in Amhara, 60.0\% and Oromia, 77.0\%.(18) On the other hand our study shows higher findings than study done in Dabat town 23.2\% (19).

In $87.6 \%$ of children age, $<12$ months colostrum was provided. The result shows a higher proportion of findings compared to assessment done in Addis Ababa, 59.8\%, in Dabat town, $51.9 \%$ and findings in SNNPR, Amhara, and Oromia which were $45.0 \%, 50.0 \%$ and $71 \%$ respectively $(9,19,18)$ This might be increasing maternal awareness in the importance of colostrum for the baby to grow fast and its protection against infections. The provision of colostrum to this rate in this area is an encouraging practice, thus could reduce both neonatal and infant mortality in the area.

\subsection{Exclusive Breastfeeding}

Early initiation and exclusive breastfeeding has been identified as one of the intervention to save new born lives, and reduce neonatal and infant morbidity and mortality rate. But this study shows low prevalence of exclusive breastfeeding practice. Only $34.1 \%$ mothers were breastfeeding for $0-<6$ months of age with the median duration of breastfeeding being 4 months. This figure is consistent with study result done in Addis Ababa 32\% and also consistent with 2000 EDHS, 38.0\% $(10,20)$, but lower than the EDHS 2005, 49.0\% and study result in Tigray $41.8 \%$ of children who were exclusively breastfed for $0-<6$ months $(9,17)$.The recent survey of community assessment of linkage project(used 24 hour recall), showed much higher prevalence of exclusive breast feeding, $81.0 \%, 71.0 \%$ and $62.0 \%$ in Amara, Oromiya and SNNP respectively (18). This could be due the fact that community based promotion of exclusive breastfeeding in Bishoftu town is still at its early stage resulting in early introduction of complimentary feeds by mothers with the perception of breast milk inadequacy for the baby. Since 24 hour recall was used by Linkage project to obtain the information, this could overestimate the exclusive breastfeeding rate and also could be due to the availability of skilled health extension workers, government service providers and NGOs who work on IYCF program might intensively promoting optimal breastfeeding practice in those regions.

On the other hand our study showed higher prevalence of exclusive breastfeeding as compared to the practice in Jimma $7.0 \%$, and study done in Dabat $18.0 \%(15,19)$

Those who did not attend formal school practice exclusive breastfeeding more likely $(\mathrm{AOR}=3.61(1.14,10.5))$ than mothers who attend formal school. This is consistent with the study done in Addis Ababa (10), but, is inversely related with study done in Tigray, where educated mothers have better practice of exclusive breastfeeding practice (17), The reason for this could be, those who did not attend formal school might stay at home and have access on practice of proper timing of exclusive breastfeeding practice than have formal education or, even though educated once have a better knowledge about the benefits of breastfeeding compared to the uneducated ones, they might still be influenced by artificial baby feeds due to pressure from neighbors, relatives and colleagues. Further to be educated means access to securing better jobs and thus better income therefore ability to purchase baby formula with ease which these group might think help their children.

Study participants who have visited health institution for postnatal care during their last birth, practiced exclusive breastfeeding significantly more than mothers who did not attend it, $(\mathrm{AOR}=2.19(1.12,4.15))$. The explanation for this can be that the availability of mothers for PNC at health facilities will give health workers an opportunity to provide information on the need to exclusively breastfeed the baby starting from immediate postnatal period.

\subsection{Optimal Breastfeeding Practice}

With regard to complementary feeding practices only 118 (21.8\%) mothers of age 6-23 months have started complementary feeding at 6 month post delivery, This is lower than the study done Amhara, 39.0\%, Oromia, 60.0\%, Madagascar, 64.0\%, and Dabat, $45.0 \%$ (18, 16, 19). Employed mothers were found to less likely practice timely complementary feeding $(\mathrm{AOR}=0.37(0.22,0.63))$ than those who were not employed. This is consistent with a study done in Tigray, where the practice of early weaning was higher among working mothers compared to house wives, $\mathrm{OR}=3.5$ (21). This could be because working mothers are forced to wean early as they lack time to feed their infants exclusively and start complementary food timely.

Those who had antenatal follow up practiced timely 
complementary feeding less likely than mothers who did not attend antenatal care, $(\mathrm{AOR}=0.45(0.22,0.85))$. The possible reasons for this could be mothers who attended antenatal care might not be informed adequately on complementary feeding by the health workers, or could be due to early resumption of work, have forced to start complementary food early.

In this study it was showed that out of the total children $(n=355)$, age $>12$ months, 269 (75.8\%) mothers have practiced an optimal duration of breastfeeding for $>12$ months. This result is consistent with the study done in Jimma town, $77.0 \%$, but lower than the finding of Dabat town, $90 \%$, mothers have breastfed for the same duration of time $(15,19$,).However, comparatively it suffices to say a better practice as optimum breastfeeding prevents children against chronic nutritional problems including stunting.

The finding in this study showed that only $48(8.8 \%)$ mothers have good practice of breastfeeding. This finding is lower than the study done in Jimma town which showed, $28 \%$ of mothers have good general practice of breastfeeding (15). The ever growing commercial feeding styles and the promotion of baby formula by different firms through different Medias might have played a greater role in the introduction of additional feeds which leads to early weaning styles.

Working mothers were found to have less practice of optimal breastfeeding $(\mathrm{AOR}=0.28(0.11,0.74))$ than mothers who stayed at home. The possible reason could be, employed mothers lack time and working outside in turn creates difficulties to practice optimal breastfeeding. Those with the income of less than 100 ETB had three times good practice of optimal breastfeeding as compared to mothers who earn $>500$ ETB $(\mathrm{OR}=3.11(1.11,8.74))$. This could be those with low income might not afford to feed their children other than breast milk, and also could be due to lack of job opportunities, thus might spend more time with their children at home creating a better chance of breastfeeding.

Women who use artificial breast milk substitutes are more likely to use sick days to care for their ill children and are less productive at work than women who follow recommended breastfeeding ( 22) Artificial feeding is associated with a substantial environmental burden, generating waste from the use of bottles and teats, the transportation of commercial breast milk substitutes and refuses from its packaging(23) Bottle feeding also shortens the period of postpartum amenorrhea and increases the risk of pregnancy (9). This study showed that about $38.0 \%$ children of 0-23 months were bottle-fed. This is higher than the study done in Tigray, $20.0 \%$ of children,$<1$ year were bottle-fed (17),but very low than study done in Addis Ababa, $438(77.7 \%)$ infants bottle fed (10).

According to 2005 EDHS, bottle feeding with nipple is not widespread in Ethiopia. However, the proportion of children who are bottle-fed rises from $8.0 \%$ among children age $<2$ months to $19.0 \%$ among children age of 6-8 months, after which it declines gradually to $8.0 \%$ among children $18-35$ months of age (9), and study done by linkages project in Amhara, Oromia and SNNP regions, showed that, 2.0\%,
$15.0 \%$, and $7.0 \%$ children age $0-<6$ months bottle-fed, respectively (18). This shows bottle feeding practice is declining significantly.

PNC follow up is one of the factors identified in this study as determinants of maternal knowledge on advantages of breastfeeding. Those with Postnatal attendance of health facilities were more likely to have sufficient knowledge compared with those who didn't attend. PNC is one important health services where mothers receive relevant health information and it is the right time when mothers are able to decide future optimal infant/child feeding practice and a period for safe motherhood as well. But this was not the case for the mothers under this study, where only about half of them $(54.4 \%)$ are informed about the benefit of breastfeeding.

In this study, prevalence of postnatal care within 45 days after delivery is $69.7 \%$. This is higher than the finding of Addis Ababa where about $49.0 \%$ mothers utilize postnatal care timely.

Nationally it is extremely low; it is only $1.3 \%$ from $3-41$ days attending postnatal care (9).In our study PNC is an important predictor of actual practice of exclusive breastfeeding. However, the prevalence of exclusive breastfeeding is very low calling for emphasis to be given by government as well as private health sectors to promote optimal breastfeeding practice at ANC, $\mathrm{PNC}$ and other $\mathrm{MCH}$ activities.

\section{Conclusion}

- Maternal knowledge on the benefit of breastfeeding both to the mother, child and family in the study area is insufficient. Further knowledge is dependent whether the mother attended PNC or not.

- Contraceptive benefits of exclusive breastfeeding and the criteria of LAM method is also significantly low.

- Though breastfeeding is a common practice in Bishoftu town, the rate of optimum breastfeeding remains to be very low.

- The level of exclusive breastfeeding practice as compared to other areas such as Amhara, SNNPR and Tigray is low as well.

- It can be concluded that employed mothers in formal sector as compared to those staying at home are practicing a low rate of exclusive breastfeeding

- ANC, birth place \& PNC established the most important predicators for exclusive breastfeeding and knowledge of mothers on advantages of breastfeeding, respectively.

\section{Acknowledgement}

I would also like to thank the Addis Ababa University, Faculty of Medicine, School of Public Health for all assistances including financial coverage. My thanks also extend to Bishoftu town administration office, the entire kebele administrators and the Bishoftu woreda health office for their dearest support in all aspects of the data collection 
process. Last but not least, my gratitude goes to the data collectors, mothers who participated in this study.

\section{References}

[1] American Academy of Pediatrics, Work group on breastfeeding. Breastfeeding and the use of human milk. Pediatrics 1997; 100: 1035-39

[2] Newburg DS, Peterson JA, Ruiz-palacios GM et al. Role of human milk lactadherin in protection against symptoms rotavirus infection. Lancet 1998; 351:116-1164.

[3] Woolridge MW, Phil D, Baum JD. Recent advances in breastfeeding. Alta paediatric JJPn 1993; 35: 1-2

[4] Peters E, Wehkamp K.H, Felberbaum RE. et al. Breastfeeding duration is determined by only few factors. European journal of Public Health, 2005; 16(2):162-7

[5] Foo LL, Quek AJA et al. Breatfeeding prevalence and practices among Singaporean, Chinese, Malysia and India mothers, Health Promotion International 2005; 20(3): 229-37

[6] World Health Organization, Division of Family Health, The prevalence and duration of breastfeeding in Urban population of Chandigrh during a decade, Indian pediator 1987;24: 879887

[7] Plan of action of the National Control of Diarrheal Diseases program, MOH, Ethiopia, 1990-1992

[8] Essential Nutrition Actions to Improve the Nutrition of Women and Children in Ethiopia, including under situations of Emergencies and HIV/AIDS; Training Manual, 2004

[9] 2005 Ethiopia Demographic and Health Survey, Central Statistical Authority, Addis Ababa, 2006

[10] Keltsela T, kebede D. Pattern of feeding Infants in Addis Ababa, Ethiopia. J. health Dev. 10(1):133-143, Ethiopia, 1996

[11] World Health Organization. Global Strategy for Infant and Young Child Feeding. WHO, Geneva, 2002. WHA55/2002/REC/1.

[12] Black R E et al. Maternal and Child Undernutrition. Global and regional exposures and health consequences. Lancet 2008; $371: 243-60$
[13] Federal Ministry of Health; Family Health Department. National Strategy for Infant and Young Child Feeding. April, 2004, Ethiopia

[14] Health and Health Related indicators 2003/04. Planning and Programming Department, FMOH, Ethiopia, December 2004.

[15] Biruk K.T. The status of breastfeeding among mothers of children aged less than two years and implication for the occurrence of acute diarrhea, Masters Thesis, Ethiopia, 2002

[16] www.internationalbreastfeedingjournal.com, 2003

[17] Girmatsion Fisseha, Gebremeskel Miruts, Mulu Tekie, Abraha W/Michael, Dejen Yemane, Tesfay Gerezigiher. Predictors of Timing of First Antenatal Care Booking at Public Health Centers in Mekelle City, Northern Ethiopia. Journal of Gynecology and Obstetrics. Vol. 3, No. 3, 2015, pp. 55-60. doi: 10.11648/j.jgo.20150303.13

[18] Getachew G: Feeding profile and diarrheal morbidity among 7-12 month infants in Tigray, Master's Thesis, Ethiopia, 2006

[19] Community Assessment in selected Wordas, in Amhara, Oromia and SNNP regions; ESHA, Ethiopia, 2006.

[20] EPHA, Abstract 11, Assessment of Infant and Young Child Feeding Practice in Dabat town, North West Ethiopia, 2006

[21] 2000 Ethiopia Demographic and Health Survey, Central Statistical Authority, Addis Ababa, May 2001.

[22] Crystal L. Patil. et al. Maternal knowledge, infant feeding practices in rural Tanzania, Department of Anthropology, University of Toronto, Canada, 2004

[23] Kull I, Almquist C, Lilja G, et al. Breastfeeding reduces the risk of asthma during the first 4 years of life. J Allergy Clin Immunol (2004) 114: 755-60 [Web of Science] [Medlin]

[24] Landbok MH. Breastfeeding as a woman's issue: conclusions and consensus, complementary concerns, and next actions Int J Gynaecol Obstet 1994; 47 (Suppl) S SI-S 61 (accessed 28 July 2009)

[25] Disha Khanna, Ankush Sachdeva, Battered Woman Syndrome: Its Repercussions and Implications on Women of the Present Era, International Journal of Clinical and Experimental Medical Sciences. Vol. 1, No. 2, 2015, pp. 7-10. doi:10.11648/j.ijcems.20150102.11 